IRENA MAMCZAK-GADKOWSKA

(Uniwersytet im. Adama Mickiewicza w Poznaniu, Instytut Historii)

\title{
AKTEN DER REICHSUNIVERSITÄT POSEN IN DEN ARCHIVBESTÄNDEN DER ADAM-MICKIEWICZ-UNIVERSITÄT
}

Die Reichsuniversität Posen war eine der „nationalsozialistischen“ Musteruniversitäten, auch Reichsuniversitäten genannt, die in den im Zweiten Weltkrieg ins Reich eingegliederten Gebieten entstanden waren ${ }^{1}$. Neben den Universitäten in Königsberg, Prag

${ }^{1}$ Die Organisation und Tätigkeit der Reichsuniversität Posen galt als Forschungsgegenstand. Siehe u.a.: B. Piotrowski, $W$ stużbie rasizmu i bezprawia. „Uniwersytet Rzeszy“ w Poznaniu 1941-1945 [Im Dienste des Rassismus und des Unrechts. Die „Reichsuniversität" Posen 1941-194], (1984); auch: ders., Die Rolle der "Reichsuniversitäten“ in der Politik und Wissenschaft des hitlerfaschistischen Deutschlands, in: Universities during World War II. Materials of the International Symposium held at the Jagiellonian University on the 40 Anniversary of „Sonderaktion Krakau“, ed. by J. Baszko, I. Połczyńska, (1984); F. Paprocki, K.M. Pospieszalski, „Reichsuniversität Posen“, „Przegląd Zachodni“, 12, 1956, B. 2, 5-8, (1958), S. 275-299; S. Różycki, Państwowy Uniwersytet Niemiecki w Poznaniu 1941-1945 [Die Reichsuniversität Posen, 1941-1945], „Nowiny Lekarskie“, $3 / 4$ (1945); H. Szołdrska, Walka z kultura polską. Uniwersytet Poznański podczas okupacji [Kampf gegen die polnische Kultur. Die Posener Uni- 
und Breslau sollte auch sie die Rolle des „Zentrums der östlichen Reichsgebiete "übernehmen, die als deutscher Kulturraum galten. Allerdings war die Reichsuniversität Posen nicht die einzige Hochschule, die während des Zweiten Weltkrieges in den polnischen Gebieten tätig war.

Im sogenannten Generalgouverment, das die nicht ins Reich eingegliederten Gebiete umfaßte, entstanden 1940 außer der Reichsuniversität Posen zwei weitere wissenschaftliche Einrichtungen: das Institut für Deutsche Ostforschung mit Sitz in Krakau und die Landwirtschaftliche Forschungsanstalt Puławy. Gescheitert sind dagegen die politischen Bemühungen des Generalgouverneurs Hans Frank in Berlin, eine nationalsozialistische Hochschule auf Basis der Jagellonen-Universität zu errichten ${ }^{2}$. Die ökonomischen Grundlagen der meisten Institutionen dieser Art bauten auf dem Vermögen polnischer wissenschaftlicher Einrichtungen auf ${ }^{3}$.

Die Reichsuniversität Posen trat an die Stelle der 1919 gegründeten Posener Universität. Beim Ausbruch des Zweiten Weltkrieges hatte die Posener Universität zwar eine lediglich 20-jährige Geschichte, sie entwickelte sich jedoch sehr lebhaft und verfügte damals über bedeutende wissenschaftliche Errungenschaften,

versität während der Okkupation], (1948); J. Kalisch, G. Voigt, ReichuniversitätPosen, in: Juni 1941. Beiträge zur Geschichte des hitlerfaschistischen Überfalls auf die Sowjetunion, hg. v. A. Anderle, W. Basler, (1961), S. 188-206; C. Łuczak, Uniwersytet Poznański w latach okupacji [Die Posener Universität in den Jahren der Okkupation], in: Dzieje Uniwersytetu im. Adama Mickiewicza 1919-1969, [hg. v. Z. Grot], (1972); M. Banasiewicz, Polityka naukowa i oświatowa hitlerowskich Niemiec na ziemiach polskich "wcielonych" do Trzeciej Rzeszy w okresie okupacji (1939-1945) [Die Wissenschafts - und Bildungspolitik des Hitlerdeutschlands auf den ins Dritte Reich „eingegliederten“ polnischen Gebieten während der Okkupation (1939-1945)], (1980).

2 Piotrowski, $W$ stużbie, S. 21, 31.

3 Paprocki, Pospieszalski, „Reichsuniversität Posen“, S. 275. 
insbesondere im Bereich der „Ostforschung“. Schon vor dem Krieg stießen die Ergebnisse dieser Forschung auf besonderes Interesse der deutschen Regierung, da die Posener Universität nach Ansicht der Deutschen als antideutsch galt. Wie erhaltene Archivbestände belegen, sollte die geplante Reichsuniversität „eine Hochburg deutscher Kulturleistung und ein Bollwerk gegenüber dem Polentum“ werden .

Die Vorbereitungen zur Gründung der Reichsuniversität Posen begannen schon im September 1939. Nach Kriegsausbruch vernichteten die Deutschen allmählich die wissenschaftlichen Bestände der polnischen Universität in Posen und verhafteten deren Professoren und Studenten, von denen einige starben und andere aus der Stadt vertrieben wurden. Trotz langer Vorbereitungen entstand die Reichsuniversität Posen erst im April 1941. Die festliche Einweihung sollte am 20. April - an dem Geburtstag Hitlers - stattfinden, was allerdings nicht gelang und die Universität schließlich eine Woche später, am 27. April 1941, eröffnet wurde. Der Staatsakt hatte einen sehr feierlichen Charakter unter anderem wurden eine Errichtungsurkunde der Reichsuniversität angefertigt sowie feierliche Poststempel und Medaillen geprägt $^{5}$. An der Feierlichkeit beteiligten sich die Vertreter der obersten politischen und militärischen Befehlshaber sowie Rektoren der Universitäten im Dritten Reich.

Die Organisationsstruktur der Reichsuniversität wuchs aus gesonderten Vorschriften hervor, die für alle Reichsuniversitäten galten. Geleitet wurde sie von einem durch den Reichsminister

4 Archiwum Uniwersytetu im. Adama Mickiewicza w Poznaniu [Archiv der Adam-Mickiewicz-Universität zu Poznań] (künftig zitiert als: A UAM), Akten der Reichsuniversität Posen, Sign. 143/73 (Bericht von Hans Streit an den Reichsbildungsminister vom 6. Januar 1940); Piotrowski, W służbie, S. 32.

${ }^{5}$ Die Gründung der Reichsuniversität Posen. Am Geburtstag des Führers 1941, (1941). 
für Wissenschaft, Erziehung und Volksbildung ernannten Rektor. Zum ersten Rektor der Reichsuniversität Posen wurde Peter Johann Carstens, Professor für Tierzucht und Haustiergenetik aus der landwirtschaftlichen Mittel- und Hochschule in Hohenheim bei Stuttgart ${ }^{6}$ gewählt. Prorektor wurde Geographieprofessor Walter Geisler 71944 wurde Professor Carstens in seiner Funktion als Rektor durch den Wirtschaftsprofessor Otto Hummel ersetzt. Neben dem Rektor amtierte ein kommissarischer Kurator, der SS-Sturmbannführer Dr. Hans Streit. Der Senat war ein beratendes Organ des Rektors.

Ursprünglich sollte die Reichsuniversität Posen Kurse in fünf Fakultäten anbieten: der Philosophischen Fakultät, Naturwissenschaftlichen Fakultät, Rechts-, Staats- und Wirtschaftswissenschaftlichen Fakultät, Medizinischen Fakultät und Landwirtschaftlichen Fakultät. Nicht alle von ihnen nahmen den Lehrbetrieb gleichzeitig auf. Einige Fakultäten, Institute und wissenschaftliche Einrichtungen wurden allmählich je nach Zunahme der Zahl der Lehrenden und des Kriegsbedarfs des Dritten Reiches aufgebaut. Geplant wurde, dass insgesamt 80 wissenschaftliche Mitarbeiter der Reichsuniversität im Durchschnitt 600 Studenten unterrichten würden ${ }^{8}$. Im ersten Semester 1941 nahmen nur 49 Professoren und Dozenten ihre Lehrtätigkeit auf, allerdings stieg diese Zahl kontinuierlich. Im Sommersemester 1944/1945 zählte die Rechts-, Staats- und Wirtschaftswissenschaftliche Fakultät schon 12 Professoren, einen Dozenten und 10 Lehrbeauftragte, die Medizinische Fakultät 9 Professoren und 2 Dozenten, die Philosophische Fakultät 26 Professoren, 5 Dozenten, 3 Lehrbeauftragte und 7 Lektoren, die Naturwissenschaftliche Fakultät 22 Professoren,

\footnotetext{
6 A UAM, Reichsuniversität Posen, Sign. 78/329.

7 Ebenda, Sign. 78/224-225.

8 Piotrowski, $W$ służbie, S. 36.
} 
8 Dozenten und einen Lehrbeauftragten und die Landwirtschaftliche Fakultät 16 Professoren, 7 Dozenten und 2 Lehrbeauftragte. Insgesamt lehrten an der Reichsuniversität Posen am Ende ihres Bestehens 131 Wissenschaftler, wobei zu betonen ist, dass viele von ihnen, häufig über ein Drittel, in der Wehrmacht dienten?.

Die Dozentenschaft der Reichsuniversität war verhältnismäßig jung, wobei die meisten Professoren ihre Titel eben an der Reichsuniversität in Posen erhielten. Gewählt wurden die Lehrkräfte weniger aufgrund ihrer wissenschaftlichen Kompetenzen, sondern in erster Linie durch das Prisma des nationalsozialistischen Interesses ${ }^{10}$. Bei der Wahl der wissenschaftlichen Mitarbeiter der neuen Universität spielten die 119 in Posen eingetroffenen Wissenschaftler aus den Baltenländern eine wichtige Rolle, darunter Dr. Reinhold Wittram, der spätere Dekan der Philosophischen Fakultät. Sie kamen hauptsächlich aus dem Johann-GottfriedHerder-Institut in Riga und dem wissenschaftlichen Institut in Dorpat. Die wichtigsten Lehrstühle an der Reichsuniversität wurden von deutschen Professoren aus dem Reich besetzt.

Die Zahl der Studierenden an der Reichsuniversität Posen blieb schwankend und von der Situation an der Front abhängig. Die meisten Studenten stammten aus Deutschland, ein Teil stellten die sogenannten Baltendeutschen, einige wiederum waren Volksdeutsche sowie ein paar Norweger ${ }^{11}$. Für die studierenden Kriegsteilnehmer wurde ein Fernstudium eingerichtet. 1941 studierten an der Reichsuniversität Posen 172 Studenten, 1943 waren es 755 (darunter 60 Prozent Studentinnen) und 1944 stieg diese Zahl auf 1228 (darunter 231 beurlaubte Studenten). In den

9 Ebenda, S. 36 u. 44; Paprocki, Pospieszalski, „Reichsuniversität Posen“, S. 282-283.

10 Paprocki, Pospieszalski, „Reichsuniversität Posen“, S. 282-283.

11 Piotrowski, $W$ służbie, S. 45. 
Semestern 1944/1945 lag die Studentenzahl bei 300 Personen, allerdings fanden die Lehrveranstaltungen nicht mehr statt ${ }^{12}$.

Nach der Befreiung Posens im Januar 1945 wurden die erhaltenen Akten der Reichsuniversität von den Mitarbeitern des in den Kämpfen um Posen zerstörten Staatsarchivs in Posen sichergestellt, wo sie in ein Verzeichnis aufgenommen und bis 1958 aufbewahrt wurden. In den Jahren 1958-1964 überreichte das Staatsarchiv in Posen die Akten der Reichsuniversität an das 1953 gegründete Archiv der Adam-Mickiewicz-Universität zu Poznań (künftig: UAM). Die größte Aktenpartie, insgesamt 438 Archiveinheiten mit der Signatur 78, wurde an die UAM am 15. März 1958 überwiesen. In den 1960er Jahren übernahm das Archiv der UAM vom Staatsarchiv in Posen zwei letzte Aktenbestände der Reichsuniversität: Am 20. April 1962 waren es 146 Archiveinheiten mit der Signatur 143 und am 9. Januar 19646 Archiveinheiten mit der Signatur 206. Insgesamt zählen die Akten der Reichsuniversität Posen 592 Archiveinheiten, was ca. 10 laufenden Metern ${ }^{13}$ von Akten entspricht. Der chronologische Umfang des Archivmaterials der Reichsuniversität Posen bezieht sich grundsätzlich auf die Jahre 1941-1944/1945, wobei sich darunter auch ältere Schriftstücke befinden, z.B. Informationen über die Professoren der Posener Universität, ein Verzeichnis aller Bediensteten der Posener Universität zum 1. April 1939, eine Auflistung aller Doktorarbeiten an der Philosophischen Fakultät der Posener Universität in der Zeit zwischen dem Ersten und Zweiten Weltkrieg ${ }^{14}$, sowie Archivalien aus den Jahren 1939-1941, als die Eröffnung der Reichsuniversität Posen vorbereitet wurde.

12 Ebenda, S. 44-45.

13 A UAM, Reichsuniversität Posen, Sign. 78/1-431, 143/1-146, 206/1-15.

14 Ebenda, Sign. 206/13-14. 
In den Akten der Reichsuniversität Posen sind die Archivalien des Rektors am zahlreichsten vertreten ${ }^{15}$. Darunter befinden sich viele amtliche Schreiben des Rektors an die obersten Machthaber des Dritten Reiches und des Warthegaus, z.B. an das Reichsministerium für Bildung, das Reichspropagandaamt (NSDAP) und das Amt des Gauleiters vom Reichsgau Warthegau ${ }^{16}$. Zum wesentlichen Teil der Rektorakten gehören Vorschriften und Verordnungen des Bildungsministers über Organisation von Universitäten und Hochschulen des Dritten Reiches aus den Jahren 1934-1944, Rundschreiben und Anordnungen des Rektors, Schriftstücke zur Studienordnung (Aufnahme, Prüfungen, Studienjahraufteilung), darunter auch zur Zulassung zum Hochschulstudium, ,jüdischer Einwohner" sowie zur Vorbeugung von Überfüllung an Hochschulen ${ }^{17}$. Von Bedeutung ist auch der Schriftverkehr des Rektors mit der Reichsstiftung für Deutsche Ostforschung über Zuschüsse für wissenschaftliche Forschungen sowie Akten der Stiftung und der von ihr geführten Forschungspolitik ${ }^{18}$.

Unter den Schriftstücken des Rektors befindet sich auch seine Korrespondenz mit den Fakultäten und Instituten, die fachliche, finanzielle und personelle Angelegenheiten betrifft sowie der Schriftwechsel mit wissenschaftlichen Instituten anderer Universitäten, z.B. mit dem Direktor des Rasseninstituts der Universität in Tübingen über die Errichtung des Lehrstuhls für Rasse- und Wissenschaftsfragen sowie der Schriftverkehr mit befreundeten Ländern bezüglich der wissenschaftlichen Hilfe und Zusammenarbeit ${ }^{19}$.

15 Ebenda, Sign. 78/1-8, 78/211-4, 78/17, 78/24/7, 78/58, 78/67, 78/137, 78/266-279, 78/283-287, 78/296-299, 78/302-304.

16 Ebenda, Sign. 78/1-3.

17 Ebenda, Sign. 78/58.

18 Ebenda, Sign. 78/21/1-4.

19 Ebenda, Sign. 78/24/7, 78/67. 
Eine weitere Gruppe bilden Akten, die den Lehrkörper und die Studenten betreffen. Diese beinhalten Schreiben, Gutachten, Unterlagen zu Dienst- und Studienreisen sowie Praktika, zu Gehältern der Beamten und Mitarbeiter und zur Immatrikulation ausländischer Studenten. Des Weiteren umfassen die Schriftstücke zahlreiche Einladungen, Bekanntmachungen und Informationen über die an der Reichsuniversität Posen angebotenen Gastvorträge.

Unter den Archiveinheiten befinden sich auch mehrere Akten des Reichsuniversitätskurators Dr. Hans Streit ${ }^{20}$, wie auch seine Verordnungen, Korrespondenzen mit den Befehlshabern des Dritten Reiches, z.B. mit dem Reichsbildungsminister Bernhard Rust sowie dem Gauleiter vom Warthegau Arthur Greiser, dem Reichsuniversitätsrektor, wie auch Pläne, Briefe und übrige Schreiben des Kurators.

Zahlreich vertreten sind in den Reichsuniversitätsbeständen auch Akten, die die wissenschaftlichen Mitarbeiter und Angestellten betreffen, wie z.B. Verzeichnisse eingestellter Wissenschaftler, Lektoren, Hilfsmitarbeiter und Angestellter ${ }^{21}$. Eine separate Gruppe bildet der Schriftverkehr über die Einstellung der Dozentenschaft an der Reichsuniversität, darunter die Einstellung von Baltendeutschen, sowie die Persönlichkeitsbeschreibung der Bewerber ${ }^{22}$. Nur sehr wenige Akten beziehen sich auf wissenschaftliche (darunter auch experimentelle) Forschungsarbeiten ${ }^{23}$. Erhalten geblieben sind Akten über soziale Umstände der Bedien-

20 Ebenda, Sign. 78/24/1, 78/56, 78/100-101, 143/62-63, 143/68, 143/72, 143/85, 143/91, 143/94, 143/146.

${ }^{21}$ Ebenda, Sign. 78/9, 78/11-13, 78/24/5-6, 78/31, 78/61, 78/66/2, 78/68-70, 78/98, 78/410, 78/, 78/415-418, 143/59, 143/71, 143/75-76, 143/80, 143/95, 143/140, 143/142, 206/14-15.

22 Ebenda, Sign. 78/98, 78/429.

23 Ebenda, Sign. 78/417. 
steten, und zwar über Wohnangelegenheiten, Kinderzuschüsse für Mitarbeiter der Reichsuniversität sowie Akten pensionierter Mitarbeiter. Separat geordnet wurden Akten der Dozentenschaft sowie der wissenschaftlichen Lehrbetriebe ${ }^{24}$.

Interessant für die wissenschaftliche Erforschung der Reichsuniversität sind die Personalakten, die in den Beständen der Reichsuniversität Posen vorzufinden waren. Ein von den Archivmitarbeitern der UAM zusammengestelltes Namensverzeichnis derjeniger Personen, deren Personalmappen im Archiv gefunden wurden, umfaßt über 100 Namen $^{25}$. Unabhängig davon blieben

24 Ebenda, Sign. 143/75-76.

25 Ebenda. In den Akten der Reichsuniversität Posen blieben Personalakten folgender Personen erhalten: Leonid Arbusow, Horst Bartholomeyczik, Erich Becker, Borris Ballenoschkin (Habilitationsschrift), Heimo Bielenstein, Konrad Bittner, Uwe Timm Bödewadt, Karl Boekholt, Maximilian Braun, Reinhold Brenneisen, Hans Brockmann, Friedrich Brückner, Erich Buchholz, Peter Carstens, Werner Contze, Friedrich Crome, Friedrich Dahms, Max Duering, Werner Döring, Władysław Drzewiecki, Christian Eckle, Friedrich Eggers, Kurt Eggers, M.J. Eggers, Egon Eltzberg, Albert Erhardt, Günter Feldmann, Erich Fischbach, Erich Fischback, Theodor Förster, Herbert Frank, Walter Freymann, Karl Friedrichs, Heinz Fritz, Walter Geisler, Amulf Gnam, Theodor Goodmann, Walter Gross, Erich Heidsieck, Manfred Hellmann, Karl Peter Heyser, Heinz Hildebrandt, Rudolf Hippius, Heinz Holfelder, Heinz Ischeryt, Maximilian Ittenbach, Josef Jabloka, Martin Jahn, Heinz Johannes, Andreas Junga, Eduard Justi, Adolf Kaller, Alois Kern, Hermann Kestner, Rolf Kisch, Andreas Kowalow, Karl Kunowski, Kurt Legenheim, Kurt Lange, Kurt Langhammer, Gerard Lemcke, Ulrich Lementy, Nicolai Lemke, Peter Liennemann, Rudolf Lochner, Georg Lockot, Werner Lohmann, Herbert Ludat, Lutz Mackensen, Edmund Majkowski, Eugenie Marga Mantey, Johannes Marguardt, Erika Mayer, Hubert Mayer, Walter Meder, Karl Heinz Meewes, Boris Meissner, Gerhard Mende, Leon Miller, Edmund Mudrak, Emil Müller, Villy Müller, Willy Neumann, Maximilian Ott, Rudolph Otto, Peter Paulsen, Ernst Petersen, Otto Petersen, Kurt Petrich, Karl Pfeiffer, Therese Pippan, Albert Ponsold, Walter Radezich, Georg Rauch, Günther Reichenkron, Rotem Reisinger, Gertrud Reise (Koschner), Robert Schabert, Wilhelm Schalt, Else 
auch die Personalien von Mitarbeitern einzelner Fakultäten der Reichsuniversität Posen partiell bestehen ${ }^{26}$. Die diesbezüglichen vollständigsten Daten beinhalten die für einzelne Semester in den Jahren 1941-1944/1945 veröffentlichten Broschüren mit Personen- und Vorlesungsverzeichnissen der Reichsuniversität Posen ${ }^{27}$.

Eine interessante Forschungsquelle sind Studentenakten. Erhalten geblieben sind die Akten der in den Jahren 1941-1944/1945 an der Reichsuniversität Posen Immatrikulierten ${ }^{28}$ und Exmatrikulierten ${ }^{29}$.

Unter den studentischen Unterlagen befindet sich auch ein umfangreicher Schriftwechsel mit dem Universitätskurator, sowie Akten zum Wehrdienst beurlaubter Studenten, Militärzuweisungen, Fragebögen des Gesundheitsdienstes, Zuweisungen zum Deutschen Roten Kreuz, Immatrikulations- und Patronatsakten von studierenden Kriegsteilnehmern sowie Akten im Krieg gefallener Studenten ${ }^{30}$. Diese Akten werden durch Unterlagen zu studentischen Finanz- und Wohnungsangelegenheiten wie Hausordnungen von Studentenwohnheimen sowie Akten bezüglich der Studentenprüfungen, insbesondere von ärztlichen Prüfungen,

Schaubert, Charlotte Schlötke, Karl Schmalfuss, Giudo Schneider, Josef Scholz, Willy Schultz, Hans Schwalm, Friedrich Schwarz, Hans Scupin, Johann Seeberg, Otto Seesemann, Jensen Sewerin, Paul Siegfried, Wilhelm Sigrid, Erich Spieshöfer, Edmund Spohr, Johannes Stamm, Günther Stark, Gerard Stavenhagen, Joachim Stobbe, Hans Stubbe, Stanisław Szukiewicz, Friedrich Tray, Georg Ch. Unruh, Friedrich Vittinghoff, Willy Wachnitz, Karl Wagner, Alfred Walter, Heinrich Walter, Erwin Wegner, Johannes Wedersann, Franz Weiler, Hienrich Weiss, Siegrid Wilhelm, Reinhard Wittram, Rudolf Zebro, Kurt Zinn.

${ }^{26}$ Ebenda, Sign. 78/9, 78/24/5, 78/410, 143/71.

27 Ebenda, Sign. 206/15/1-7.

28 Ebenda, Sign. 78/135, 78/205-209, 78/387.

29 Ebenda, Sign. 78/271.

30 Ebenda, 78/10, 78/55, 78/248-249, 78/25-258, 78/378-385, 78/392. 
ergänzt $^{31}$. Den studentischen Akten liegen wenige Studentenverzeichnisse für einige Fakultäten, eine Verordnung des Rektors über Hochschulzulassungen für ,jüdische Einwohner" sowie Personalfragebögen von Personen aus ,ehemaligen polnischen Gebieten " und von Baltendeutschstudenten ${ }^{32}$ bei. Erhalten blieben auch Umfragen, einzelne Universitätsstudienpläne sowie Unterlagen zu pflichtmäßigen Leibesübungen und körperlicher Erziehung für die Studenten während der ersten drei Semester (in den Übungsgebieten: Schwimmen, Schießen, Gymnastik, Laufen und Handball $)^{33}$.

Ein interessantes Forschungsmaterial bilden Archivalien, die akademische Einrichtungen wie NSD - ein Studentenbund der NSDAP oder NSDS-Amt für Studentinnen - betreffen. Sie enthalten Mitgliederverzeichnisse von Studierenden sowie Pläne und Einladungen zu Seminaren ${ }^{34}$. In den Akten der Reichsuniversität Posen befinden sich außerdem Vor- und Diplomarbeiten von Studenten verschiedener Fakultäten ${ }^{35}$.

Einige wenige erhaltene Archivalien zeigen die Tätigkeit einzelner Institute, Fakultäten und Anstalten der Reichsuniversität, z.B. des Instituts für Experimentalphysik ${ }^{36}$, Instituts für Anorganische Chemie und Chemische Technologie ${ }^{37}$, des Hygienischen Instituts ${ }^{38}$, der Rechts-, Staats- und Wirtschaftswissenschaftlichen Fakultät ${ }^{39}$, der Naturwissenschaftlichen Fakultät ${ }^{40}$, des Geogra-

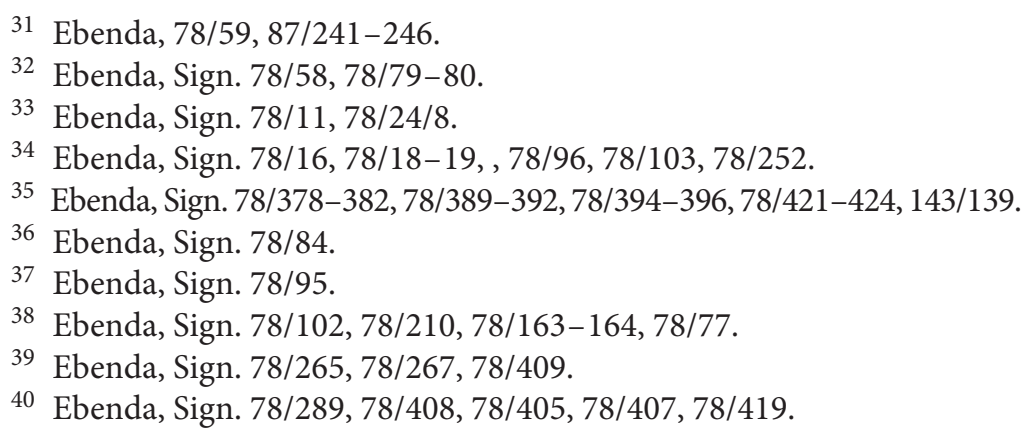


phischen Instituts ${ }^{41}$, der Versuchsgüter ${ }^{42}$ sowie auch die Finanzangelegenheiten des Zentralinstituts für Krebsforschung ${ }^{43}$. Eine umfangreichere, wenn auch nicht vollständige Unterlagensammlung betrifft die Philosophische und Medizinische Fakultät. In den Akten der Philosophischen Fakultät befinden sich Unterlagen zur Stellenbesetzung und Studentenpraktika, ein umfangreicher Briefverkehr mit Wissenschaftlern sowie Schriftstücke, die Seminare in Geschichte, in Englischer, Romanischer und Slawischer Philologie, Psychologie und Pädagogik wie auch das Indogermanische Seminar betreffen ${ }^{44}$.

Die Akten der Medizinischen Fakultät sind unter den Archivalien zahlreicher vertreten ${ }^{45}$. Darunter befinden sich in großer Zahl: der Schriftverkehr des Fakultätsdekans mit den Machthabern des Dritten Reiches, dem Gauleiter des Warthegaus sowie der Dozentenschaft und den Studierenden. Eine getrennte Aktengruppe bilden die Verordnungen des Reichsbildungsministers, Schreiben bezüglich der Übergabe von Medikamenten zu Kriegszwecken, Verordnungen über Strom- und Heizmitteleinsparungen. Wenige Archivstücke betreffen die Organisation eines neuen Sonderkrankenhauses, Verordnungen über den Einsatz von Kriegsgefangenen als Versuchspersonen bei Forschungsprojekten sowie Kriegsaufgaben für Ärzte und Studierende. Erhalten blieben auch fragmentarische Akten bezüglich der Universitätsklinik, der Dozentenschaft der Medizinischen Fakultät sowie der Verordnungen und Anforderungen bei Habilitationsschriften. Diese sind

41 Ebenda, Sign. 78/307.

42 Ebenda, Sign. 78/125.

43 Ebenda, Sign. 143/69-70.

44 Ebenda, Sign. 78/81-82, 78/85-90, 78/105, 78/117-118, 78/127-129, 78/302, 78/305, 78/406.

45 Ebenda, Sign. 78/20, 78/24/3, 78/28-29, 78/34-52, 78/59, 78/133-134, $78 / 156$. 
ergänzt durch Akten der Medizinstudenten, ärztliche Prüfungen und studentische Auslandsreisen. Die erhaltenen Akten der Medizinischen Fakultät sind nicht immer ein aussagekräftiges Forschungsmaterial, jedoch zeugt die Anzahl der Studierenden und der Dozentenschaft davon, dass diese Fakultät zu einer der wichtigsten an der Reichsuniversität Posen gehörte.

Es blieben keine Archivalien erhalten, die einen umfassenden Einblick in die Forschungsaktivitäten der Reichsuniversität Posen gewähren. Abgesehen von den oben genannten Personalakten der Dozentenschaft, die einige wissenschaftliche Aktivitäten der Reichsuniversität gewissermaßen abbilden, befinden sich im Archiv der UAM lediglich einzelne Archiveinheiten, die das Bestehen diesbezüglicher Arbeitsfelder belegen ${ }^{46}$. Dazu gehören Vorschriften über Dissertations- und Habilitationsschriften aus den Jahren 1934-1943, Akten zu ideologischen Anforderungen an die Dozentenschaft, vereinzelte Akten zu Dissertationen sowie Verfügungen des Rektors bezüglich des Widerrufs der Doktorwürde für die Dozentenschaft. Neben den studentischen Diplomarbeiten aus verschiedenen Fakultäten und einzelnen Referaten sind einige wissenschaftliche Arbeiten enthalten, z.B.: die Habilitationsschrift von Dr. Borris Bellenoschkin mit dem Titel Biologie und Klinik der Spermatozoen (Experimentale Untersuchungen am menschlichen Sperma ausserhalb des Körpers und im weiblichen Organismus) ${ }^{47}$, die Arbeit von Wolf M.H. Weisswange Untersuchungen über den Einfluss der Strahlenintensität auf den Kaninchenhoden ${ }^{48}$ sowie die Arbeit von Georg von Rauch Die Posener Akademie. Eine Volksbildungsstätte im deutschen Osten (1903-1919)49.

\footnotetext{
46 Ebenda, Sign. 78/25-27, 78/72, 78/418-419, 143/59.

47 Ebenda, Sign. 78/418, MS, K. 61.

48 Ebenda, Sign. 78/417, MS, K. 77.

49 Ebenda, Sign. 78/430, MS, K. 122.
} 
Erhalten geblieben sind in den Akten der Reichsuniversität Posen dagegen Unterlagen, welche die wissenschaftliche Arbeit der Universität mit anderen Universitäten, Hochschulen und wissenschaftlichen Stiftungen belegen. Dazu gehören in erster Linie Akten über die Kooperation der Reichsuniversität mit der Reichsstiftung für Deutsche Ostforschung, die in Posen sehr aktive Ostforschungsarbeiten betrieb ${ }^{50}$. Darunter findet sich der Schriftwechsel zwischen diesen zwei Einrichtungen, ein Verzeichnis der Arbeitsfelder sowie Akten, welche die Forschungspolitik der Stiftung widerspiegeln. Des Weiteren sind dort Archivalien vertreten, die eine Zusammenarbeit der Reichsuniversität Posen mit der 1940 zur Ausbildung von Beamten auf besetzten Gebieten gegründeten deutschen Verwaltungsakademie Wartheland belegen. In der Akademie lehrten neben Parteigenossen, Kulturräten, Betriebsleitern und Landräten auch wissenschaftliche Mitarbeiter der Reichsuniversität Posen, z.B. die Professoren E. Becker, R. Brenneisen, R. Wittram und andere ${ }^{51}$. Darüber hinaus wurde Anfang 1942 die Arbeitsgemeinschaft der Reichsuniversität Posen-Litzmannstadt tätig ${ }^{52}$. Das Geographische Institut der Reichsuniversität Posen war an dem Industriegebiet um Litzmannstadt sehr interessiert und aus seiner Initiative wurde eine "Arbeitsgemeinschaft" gegründet, die sich Organisation sowie wissenschaftliche Betreuung und Finanzierung von Forschungsarbeiten zu wirtschaftlichen, kulturellen, politischen und kommunalen Angelegenheiten von Stadt und Kreis Litzmannstadt zum Ziel setzte ${ }^{53}$.

$\mathrm{Zu}$ den wissenschaftlichen Anstalten der Reichsuniversität Posen gehörte die Universitätsbibliothek. Die Bibliothek der

50 Ebenda, Sign. 78/23/1, 78/23/2.

51 Ebenda, Sign. 143/76; Piotrowski, W stużbie, S. 148-149.

52 Ebenda, Sign. 78/85, 78/284, 78/282, 78/358.

53 Ebenda, Sign. 78/282, Piotrowski, W stużbie, S. 134. 
Posener Universität wurde von den Deutschen noch 1939, also einige Jahre vor der Entstehung der Reichsuniversität, beschlagnahmt und schließlich übernommen ${ }^{54}$. In den Akten der Reichsuniversität Posen befindet sich eine Mappe mit Unterlagen zur Organisation, Tätigkeit und zum Personalwesen der Bibliothek ${ }^{55}$.

Die Reichsuniversität Posen verfügte über keine eigenen Veröffentlichungen. Die erhaltenen Archivalien ergeben, dass die Universitätsleitung plante, noch vor Kriegsende (1944) „Jahrbücher der Reichsuniversität Posen" herauszugeben ${ }^{56}$.

Sitz der Reichsuniversität Posen waren Gebäude und Räume der Posener Universität, die von den Besatzungsmächten schon im September 1939 übernommen worden waren. Dies belegen die in den Akten der Reichsuniversität enthaltenen Unterlagen zur Vermögenslage der Reichsuniversität bei der Aufnahme des Lehrbetriebes ${ }^{57}$. Einsehbar sind Akten, die Verzeichnisse übernommener Grundstücke und Universitätsgebäude sowie genaue Lagepläne der Reichsuniversitätsfakultäten, Institute, Studentenheime, Dozentschaftsheime und der Bibliothek enthalten ${ }^{58}$. Eine separate Aktengruppe bilden Akten mit Unterlagen bezüglich des Austauschs, der Pacht und Gewinnung neuer Grundstücke für den Bau eines Universitätscampus ${ }^{59}$. Erhalten geblieben ist ferner ein umfangreicher Schriftverkehr des Reichskurators mit der

54 J. Baumgart, Biblioteka Uniwersytecka pod rządami „Reichsuniversität" [Die Universitätsbibliothek unter der Macht der „Reichsuniversität"], „Przegląd Zachodni“, 12, B. 2, 5-8 (1956), S. 300-310; auch: ders., Los bibliotek wielkopolskich w latach 1939-1945 [Das Schicksal der Bibliotheken Großpolens in den Jahren 1939-1945], „Przegląd Zachodni“, 11 (1946), S. 928-936.

55 A UAM, Sign. 78/32.

56 Ebenda, Sign. 78/201; Piotrowski, W służbie, S. 46.

57 A UAM, Reichsuniversität Posen, Sign. 206/6.

58 Ebenda, Sign. 143/103, 143/99.

59 Ebenda, Sign. 78/432, 143//104, 143/114/ 143/119, 143/128-129, 143/143, 206/5-6. 
Stadtverwaltung aus den Jahren 1940-1944, der die Verwaltung von Grundstücken und die Baupläne der Reichsuniversität betrifft ${ }^{60}$. Die erhaltenen Archivstücke belegen, dass der Campus im Stadtteil Winiary i Winogrady gebaut werden und als repräsentatives Symbol der deutschen Macht im „europäischen Osten“ gelten sollte. Geplant wurde insbesondere der Ausbau der Medizinischen Fakultät samt Kliniken und der Landwirtschaftlichen Fakultät ${ }^{61}$.

In den Akten der Reichsuniversität Posen befinden sich auch einige Archiveinheiten mit Finanzplänen und -berichten ${ }^{62}$, sowie mit manchen in der Kanzlei der Reichsuniversität eingesetzten Hilfsmitteln zur Buchführung, z.B. Tage- und Titelbücher wie auch Anordnungen und Rundschreiben zur Kanzleiführung und Organisation von Büroarbeit an deutschen Hochschulen ${ }^{63}$.

Die im Archiv der UAM aufbewahrten Akten der Reichsuniversität Posen wurden nicht ausgesondert. Sie sind ausgestattet mit einem Findbuch und einem Registerverzeichnis, das Personalakten umfaßt. An den Akten zeigen Interesse sowohl polnische Forscher als auch Wissenschaftler aus dem Ausland, darunter aus den USA.

Nicht nur das Archiv der UAM besitzt Akten zur Geschichte und Tätigkeit der Reichsuniversität Posen. Die diesbezüglichen Archivbestände werden auch in anderen Archiv- und Forschungsanstalten der Stadt Posen aufbewahrt, unter anderem im Staats-

60 Ebenda, S. 143/95-96.

61 Ebenda, Sign. 78/432, 143/103-104, 143/143; Biblioteka Uniwersytetu im. Adama Mickiewicza w Poznaniu [Bibliothek der Adam-Mickiewicz-Universität zu Poznań] (künftig zitiert als: B UAM), Archiv der Bibliothek, Sign. 279; B. Piotrowski, W służbie, S. 45-46.

62 A UAM, Reichsuniversität Posen, Sign. 143/45-51, 143/58.

63 Ebenda, Sign. 78/290-295, 143/3-5, 143/43, 143/58. 
archiv beim Reichsstatthalter im Warthegau ${ }^{64}$, im Archiv des Westinstituts ${ }^{65}$, sowie in den Handschriftbeständen der Universitätsbibliothek ${ }^{66}$ und im Archiv der Universitätsbibliothek ${ }^{67}$.

Die in dem Archivbestand der UAM aufbewahrten Archivalien, obwohl nicht vollständig und wissenschaftlich unterschiedlich relevant, sind ein interessantes Quellenmaterial für die historische Forschung mit Bezug auf die Reichsuniversität Posen. Die Forscher finden darin interessante Informationen zur Entstehung, Organisationsstruktur, zum Lehr- und Forschungsbetrieb sowie zum politisch-ideologischen Engagement der Reichsuniversität. Auch die Rolle der deutschen Hochschulen und deutschen Wissenschaftler in der Umsetzung der nationalsozialistischen Politik auf den besetzten Gebieten wird daraus ersichtlich.

64 Archiwum Państwowe w Poznaniu [Staatsarchiv in Posen], Reichsstatthalter im Warthegau, Findbuch, Nr 299.

65 Archiwum Instytutu Zachodniego w Poznaniu [Archiv des Westinstituts in Posen], Sign. I.Z. Dok. I - 22, 181, 246-249, 275, 282, 392, 679-684, $705,711,715,720,725,743,749,750-753,755,760,770,786,793,919,931$.

66 B UAM, Handschriftbeständen der Bibliothek, HS, 301 III, 1136/1-7, 1137/1-23, 1138, 1146, 1625, 1642/1-7, 1702/1-2, 2054, 2342, 2661, 2788, 2823-2824, 3032-3037, 3266, 3420, 3479, 3505, 3525, 3600, 3613, 3852.

67 B UAM, Archiv der Bibliothek, Sign. 255, 257-264, 266-274, 276-281, 2056-2059, 2105, 2107-2115. 


\section{AKTA UNIWERSYTETU RZESZY W POZNANIU W ZASOBIE ARCHIWALNYM UNIWERSYTETU IM. ADAMA MICKIEWICZA}

(STRESZCZENIE)

W artykule przedstawiono część zasobu archiwum Uniwersytetu im. Adama Mickiewicza, która obrazuje działalność dydaktyczną, naukową i polityczną poznańskiego Uniwersytetu Rzeszy (Reichsuniversität) w czasie II wojny światowej. Uniwersytet ten (działał w latach 1941-1945) był jedną z wzorcowych narodowosocjalistycznych uczelni wyższych powstałych na terenach wcielonych do Rzeszy. Został on utworzony na bazie założonego w 1919 roku Uniwersytetu Poznańskiego.

Powstałe w czasie kilkuletniej działalności tego Uniwersytetu akta, chociaż niepełne (zachowało się bowiem tylko kilkaset jednostek archiwalnych) stanowią cenny materiał źródłowy. Najliczniejszą grupę stanowią akta rektora, kuratora, akta personalne pracowników oraz studentów. Zachowane materiały nie obrazują w pełni działalności wszystkich wydziałów i instytutów tej uczelni, pokazują jednak główne kierunki jej rozwoju. Najobszerniejsza dokumentacja dotyczy Wydziału Lekarskiego i Filozoficznego. Zachowały się także nieliczne prace dyplomowe studentów oraz kilka prac naukowych. Interesujący materiał stanowią akta dotyczące współpracy poznańskiego uniwersytetu $\mathrm{z}$ innymi uczelniami wyższymi Rzeszy oraz fundacjami naukowymi.

Akta wytworzone przez Uniwersytet Rzeszy, znajdujące się w zasobie Archiwum UAM stanowią ważny materiał źródłowy do badań nad okolicznościami powstania, strukturą organizacyjną, działalnością dydaktyczną i polityczno-propagandową uczelni. Pozwalają na pokazanie roli niemieckich uczelni oraz uczonych w procesie realizacji polityki III Rzeszy na ziemiach okupowanych. 